\title{
Imposing Long-Range Ferromagnetic Order in Rare-Earth Doped Magnetic Topological-Insulator Heterostructures
}

\author{
L. B. Duffy, ${ }^{1,2}$ A. Frisk,${ }^{3}$ D. M. Burn, ${ }^{3}$ N.-J. Steinke, ${ }^{2}$ J. \\ Herrero-Martin, ${ }^{4}$ A. Ernst, ${ }^{5}, 6$ G. van der Laan, ${ }^{3}$ and T. Hesjedal ${ }^{1}$ \\ ${ }^{1}$ Clarendon Laboratory, Department of Physics, University of Oxford, \\ Parks Road, Oxford, OX1 3PU, United Kingdom \\ ${ }^{2}$ ISIS, Rutherford Appleton Laboratory, Harwell Science and Innovation Campus, \\ Science and Technology Facilities Council, Oxon OX11 0QX, United Kingdom \\ ${ }^{3}$ Magnetic Spectroscopy Group, Diamond Light Source, Didcot, OX11 ODE, United Kingdom \\ ${ }^{4}$ CELLS-Divisió Experiments, ALBA Synchrotron Light Source, \\ E-08290 Cerdanyola del Vallès, Barcelona, Catalonia, Spain \\ ${ }^{5}$ Max-Planck-Institut für Mikrostrukturphysik, Weinberg 2, 06120 Halle, Germany \\ ${ }^{6}$ Institute for Theoretical Physics, Johannes Kepler University, A-4040 Linz, Austria
}

(Dated: April 23, 2018)

\begin{abstract}
The combination of topological properties and magnetic order can lead to new quantum states and exotic physical phenomena, such as the quantum anomalous Hall (QAH) effect. The size of the magnetic gap in the topological surface states, key for the robust observation of the QAH state, scales with the magnetic moment of the doped 3D topological insulator (TI). The pioneering transition-metal doped $(\mathrm{Sb}, \mathrm{Bi})_{2}(\mathrm{Se}, \mathrm{Te})_{3}$ thin films only allow for the observation of the QAH effect up to some $100 \mathrm{mK}$, despite the much higher magnetic ordering temperatures. On the other hand, high magnetic moment materials, such as rare-earth doped $(\mathrm{Sb}, \mathrm{Bi})_{2}(\mathrm{Se}, \mathrm{Te})_{3}$ thin films, show large moments but no long-range magnetic order. Proximity coupling and interfacial effects, multiplied in artificial heterostructures, allow for the engineering of the electronic and magnetic properties. Here, we show the successful growth of high-quality $\mathrm{Dy}: \mathrm{Bi}_{2} \mathrm{Te}_{3} / \mathrm{Cr}: \mathrm{Sb}_{2} \mathrm{Te}_{3}$ thin film heterostructures. Using x-ray magnetic spectroscopy we demonstrate that high transition temperature $\mathrm{Cr}: \mathrm{Sb}_{2} \mathrm{Te}_{3}$ can introduce long-range magnetic order in high-moment $\mathrm{Dy}: \mathrm{Bi}_{2} \mathrm{Te}_{3}$ - up to a temperature of $17 \mathrm{~K}-$ in excellent agreement with first-principles calculations, which reveal the origin of the long-range magnetic order in a strong antiferromagnetic coupling between Dy and Cr magnetic moments at the interface extending over several layers. Engineered magnetic TI heterostructures may be an ideal materials platform for observing the QAH effect at liquid He temperatures and above.
\end{abstract}

PACS numbers: 75.30.Hx; 78.70.Dm; 75.50.Pp; 73.61.Ng

\section{INTRODUCTION}

The quantum anomalous Hall (QAH) effect [1-3], the final member to complete the quantum Hall trio [4], is characterized by dissipationless edge currents in the absence of an applied magnetic field, whereby the resistance is quantized in units of $h / e^{2}$. The QAH effect emerges in magnetically doped 3D topological insulators (TIs), in which time-reversal symmetry is broken and a magnetic gap opens around the Dirac point of the undoped TI. Cr-doped, and also V-doped (Sb,Bi) $)_{2} \mathrm{Te}_{3}$ have been the key material for observing the QAH effect $[5,6]$. These films have useful electronic properties, even at higher $\mathrm{Cr}$ doping levels [7-9], and the magnetic ordering temperature can be above $100 \mathrm{~K}$ without loss of crystalline quality [10]. Nevertheless, the observation of the QAH effect has been restricted to very low temperatures $(<300 \mathrm{mK})$ despite the much larger ordering temperatures of the magnetic TIs $[5,6]$, which has been ascribed to extreme Dirac-mass disorder [11]. Despite being well-behaved structurally, substitutional $\mathrm{Cr}$ doping into this materials class (replacing $\mathrm{Bi}^{3+}$ ), was found to lead to nominally divalent $\mathrm{Cr}$ and covalent bonding, thereby altering the structural and magnetic properties $[12,13]$.
In an attempt to increase the useful QAH-effect temperature, a number of doping approaches have been explored, such as co-doping with different transition metals [14], modulation doping to separate the dopant from the charge-carrying surface state layer $[15,16]$, and doping with higher magnetic moment rare earth ions $[17,18]$, to name a few. Whereas co-doping leads to further complications in this materials class [14], modulation doping with $\mathrm{Cr}$ has led to the first successful observation of the axion insulator state [19, 20]. Modulation doping of heterostructures, i.e., non-uniform doping, is a wellestablished technique in III-V semiconductors leading to ultrahigh electron mobilities [21]. It relies on the separation of the donors, which can lead to ionized impurity scattering, from the conduction paths of the carriers near the interface [22]. In this spirit, magnetic modulation doping can lead to reduced disorder in the path of the topological surface state, yet being able to open the required magnetic gap.

Another approach is to dope the TI with a higher magnetic moment dopant, such as $4 f$ rare earth elements. As the size of the magnetic gap is directly dependent on the size of the magnetic moment [23], doping with, e.g., Dy with an effective magnetic moment of $10.6 \mu_{\mathrm{B}}$ /atom could allow for lower doping concentrations. Another ad- 
vantage of rare earth dopants is that they substitute Bi iso-electronically [24], leading to less charge scattering. $\mathrm{Bi}_{2} \mathrm{Te}_{3}$ thin films have been successfully highly doped with Ho [25], Gd [24], and Dy [17], however, no ferromagnetic long-range order has been detected. Nevertheless, even in the absence of long-range ferromagnetic order, a gapped topological surface state band has been observed in Dy-doped $\mathrm{Bi}_{2} \mathrm{Te}_{3}$ [18]. A similar behavior has been found in $\mathrm{Bi}_{2-x} \mathrm{Cr}_{x} \mathrm{Se}_{3}$ thin films. There, the gap opening has been attributed to short-range ferromagnetic order resulting from inhomogeneous doping and the formation of Cr clusters [26], which could be ruled out for Dy-doped $\mathrm{Bi}_{2} \mathrm{Te}_{3}$ by high-angle annular dark field scanning transmission electron microscopy studies [18].

In order to combine the advantages of the hightemperature order in Cr-doped systems with the high magnetic moments of rare earth element-doped films, yet avoiding the respective shortcomings, we have explored magnetically doped $\mathrm{Bi}_{2} \mathrm{Te}_{3} / \mathrm{Sb}_{2} \mathrm{Te}_{3}$ heterostructures [27-29]. We demonstrate the successful growth of high quality magnetic TI heterostructures using molecular beam epitaxy (MBE). The heterostructures consist of well defined bilayers of Dy-doped $\mathrm{Bi}_{2} \mathrm{Te}_{3}$ and $\mathrm{Cr}$-doped $\mathrm{Sb}_{2} \mathrm{Te}_{3}$ as characterized by in-situ reflection high energy electron diffraction (RHEED) and x-ray diffraction (XRD). We use x-ray absorption spectroscopy (XAS) and x-ray magnetic circular dichroism (XMCD) to investigate the chemical and magnetic properties of the heterostructures, and to unambiguously determine the magnetic order of the Dy-doped layers. To separate near-surface effects, such as oxidation, from the inherent properties of the heterostructure we use both surfacesensitive total-electron-yield (TEY) detection and x-ray transmission detected by luminescence yield (LY). The clear presence of an XMCD signal at remanence suggests ferromagnetic ordering of the Dy. Isothermal magnetization curves (Arrott plots), obtained element-specifically at the Dy $M_{5}$ edge, yield ferromagnetic ordering up to $17 \mathrm{~K}$. The neighboring Cr layers show an ordering temperature of $\sim 70 \mathrm{~K}$. Using first-principles calculations, we find that while a $\mathrm{Cr}: \mathrm{Sb}_{2} \mathrm{Te}_{3}$ single-layer is ferromagnetically order, the $\mathrm{Dy}: \mathrm{Bi}_{2} \mathrm{Te}_{3}$ single-layer is paramagnetic. Surprisingly, the exchange interaction between Dy moments is positive and very strong, but only within the Bi layer between the nearest neighbors. By introducing the $\mathrm{Cr}: \mathrm{Sb}_{2} \mathrm{Te}_{3} / \mathrm{Dy}: \mathrm{Bi}_{2} \mathrm{Te}_{3}$ interface, and through hole-induced polarization, the magnetic interaction in the vicinity of the interface is enhanced, extending over several layers. This demonstrates that through engineering of the heterostructure, the high moment of the rare earth can have long-range magnetic order through the proximity to a Cr-doped TI layer.

\section{METHODS}

\section{A. Sample growth}

The thin film heterostructures were synthesized by molecular beam epitaxy (MBE) using established recipes for the $\mathrm{Dy}: \mathrm{Bi}_{2} \mathrm{Te}_{3}$ and $\mathrm{Cr}: \mathrm{Sb}_{2} \mathrm{Te}_{3}$ layers [12, 17, 30, 31]. The $c$-axis oriented films were grown on $c$-plane sapphire substrates using elemental sources in an MBE system with a base pressure of $1 \times 10^{-10}$ mbar. $\mathrm{Bi}, \mathrm{Sb}$ and $\mathrm{Te}$ (6N purity) were evaporated from standard effusion cells, while Cr (6N purity) and Dy (4N purity) were evaporated using high temperature cells. Flux ratios of $\sim 100: 10: 1$ for Te:(Sb,Bi):(Cr,Dy), as measured by an in-situ beam flux monitor (BFM), were maintained throughout the growth. First, a $\sim 3$-nm-thick undoped $\mathrm{Bi}_{2} \mathrm{Te}_{3}$ film was deposited at a substrate temperature of $200^{\circ} \mathrm{C}$, which was then annealed at a substrate temperature of $250^{\circ} \mathrm{C}$ in a background flux of Te $[17,32]$. The heterostructure was then grown on top of this layer starting with $\mathrm{Dy}: \mathrm{Bi}_{2} \mathrm{Te}_{3}$, and followed by $\mathrm{Cr}: \mathrm{Sb}_{2} \mathrm{Te}_{3}$. The heterostructures consist of $n$ bilayer repeats, whereby each bilayer is made up of equally thick Dy: $\mathrm{Bi}_{2} \mathrm{Te}_{3}$ and $\mathrm{Cr}: \mathrm{Sb}_{2} \mathrm{Te}_{3}$ layers. The layer thicknesses were calibrated by x-ray reflectivity. For surface-sensitive x-ray spectroscopy measurements, the heterostructures were capped with a layer of $\mathrm{Bi}$, which has an optimal thickness to both protect the sample from oxidation, yet allow for soft x-ray spectroscopy to be carried out.

The quality of the layers across the interfaces was monitored using in-situ reflection high-energy electron diffraction (RHEED). The RHEED patterns exhibit streaks during the growth of each layer, indicating smooth, two-dimensional layers.

\section{B. Structural characterization}

X-ray diffraction (XRD) and x-ray reflectometry (XRR) were carried out using a Bruker D8 Discover diffractometer with $\mathrm{Cu}-K \alpha_{1}$ radiation using a $\mathrm{Ge}(220)$ 2-bounce monochromator in combination with $2.5^{\circ}$ Soller slits and $0.6 \mathrm{~mm}$ beam mask. Depending on the mode, we either used $2.5^{\circ}$ Soller slits in combination with a scintillation counter for both XRD and XRR. The crystalline quality and phase of the layers in the heterostructure are determined using out-of-plane XRD (scanning along the $l$-direction). The $c$-axis lattice constants are obtained by fits to the observable $\left(\begin{array}{lll}0 & l\end{array}\right)$ order reflections (with $l$ $=3,6, \ldots)$, which are indexed according to the $\mathrm{Bi}_{2} \mathrm{Te}_{3}$ and $\mathrm{Sb}_{2} \mathrm{Te}_{3}$ unit cells. The film thicknesses reported in the paper, as well as the RMS (root-mean square) surface roughnesses, which were found to be between $1.0 \mathrm{~nm}$ $\left(\mathrm{Dy}: \mathrm{Bi}_{2} \mathrm{Te}_{3}\right)$ and $1.5 \mathrm{~nm}\left(\mathrm{Cr}: \mathrm{Sb}_{2} \mathrm{Te}_{3}\right)$, were obtained using XRR. The film stoichiometries were determined to be $\mathrm{Dy}_{0.72} \mathrm{Bi}_{1.28} \mathrm{Te}_{3}$ and $\mathrm{Cr}_{0.42} \mathrm{Sb}_{1.58} \mathrm{Te}_{3}$. 


\section{X-ray spectroscopy}

X-ray absorption spectroscopy (XAS) and x-ray magnetic circular dichroism (XMCD) measurements were performed on beamline I10 at the Diamond Light Source (Didcot, UK) and beamline 29 (BOREAS) at ALBA (Barcelona, Spain). XAS was measured at the $\mathrm{Cr} L_{2,3}$, Dy $M_{4,5}$, and $\mathrm{Sb} M_{4,5}$ edges in both total-electron-yield (TEY) mode, providing surface-sensitive measurements with a probing depth of 3-5 nm, and fluorescence-yield (FY) mode, which is more bulk sensitive [33, 34]. TEY worked better for the $\mathrm{Cr}$, as it is contained in the top layer, while FY worked better for Dy, which is in the second layer. On the other hand, luminescence-yield (LY) mode probes the entire stack of the heterostructure. In LY, the transmitted x-rays which are not absorbed in the sample stack give rise to x-ray excited optical luminescence in the sapphire substrate. The emitted optical photons exit through a hole in the back of the sample holder and are detected by a photodiode [see inset of Fig. 3(a)].

Prior to the measurements, the samples were fieldcooled in a field of $6 \mathrm{~T}$. The applied magnetic field is always along the beam direction. The TEY measurements were carried out with the sample at an angle of $54.7^{\circ}$, i.e., the magic angle, at which the anisotropic contributions to the spin and orbital moments disappear $[35,36]$, to accommodate for the expected perpendicular magnetization direction of the $\mathrm{Cr}_{\mathrm{Sb}} \mathrm{Sb}_{2} \mathrm{Te}_{3}$ (easy axis out-of-plane [31]). Single-layer $\mathrm{Dy}: \mathrm{Bi}_{2} \mathrm{Te}_{3}$, on the other hand, are expected to have the easy axis in-plane [17]. The measurements in LY were carried out with the x-ray beam at $21^{\circ}$ incidence in order to optimize the LY from the sample and substrate combination. The XAS spectra were calculated by taking the negative logarithm of the LY intensity after normalizing it by the incident beam intensity.

The XMCD was obtained by taking the difference between XAS spectra with the helicity vector antiparallel and parallel along the beam direction, respectively.

\section{Theoretical calculations}

First-principles calculations were performed using a self-consistent multiple scattering Green function within the density functional theory in a generalized gradient approximation [37]. The method specially designed for semi-infinite systems such as surfaces, interfaces and heterostructures [38]. Relativistic effects were taken into account by solving the full relativistic Dirac equation within a full potential approximation [39]. The doping was simulated within a coherent approximation [40] within the multiple scattering theory [41]. Strongly localized Dy $4 f$ states were treated within a GGA $+\mathrm{U}$ approximation [42] using the Hubbard parameter $U=6 \mathrm{eV}$. The exchange interaction between magnetic moments was estimated via the magnetic force theorem as it is formulated within the multiple scattering theory [43]. The Curie temperature was determined using a random phase approximation for the magnonic Green function and temperature dependent magnetization utilizing the Tyablikov decoupling [44]. The crystalline structure and the stoichiometry of studied systems was adopted from the current experiment.

\section{RESULTS}

\section{A. Synthesis of the magnetic TI heterostructures}

The heterostructures consisting of Dy-doped $\mathrm{Bi}_{2} \mathrm{Te}_{3}$ and Cr-doped $\mathrm{Sb}_{2} \mathrm{Te}_{3}$ bilayers were grown by $\mathrm{MBE}$ on $c$-plane sapphire $\left(\mathrm{Al}_{2} \mathrm{O}_{3}\right.$ [0001]) substrates. First, a 3nm-thick $\mathrm{Bi}_{2} \mathrm{Te}_{3}$ seed layer was deposited using our established two-step method [17, 32], followed by alternating sequences of 4-nm-thick $\mathrm{Dy}: \mathrm{Bi}_{2} \mathrm{Te}_{3}$ and $\mathrm{Cr}: \mathrm{Sb}_{2} \mathrm{Te}_{3}$ layers at a substrate temperature of $250^{\circ} \mathrm{C}[12,17,30$, 31]. For the magnetic spectroscopy studies, we chose a sample with 10 bilayer repeats. For protection against oxidation, the samples were capped with a Bi layer.

In-situ reflection high-energy electron diffraction (RHEED) was used to observe the quality of the surface of each deposited layer as shown in Fig. 1a,b. The RHEED patterns exhibit clear streaks, indicative of smooth surfaces. The two characteristic RHEED pat-

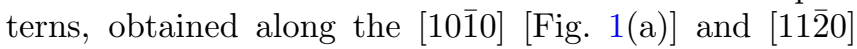
[Fig. 1(b)] azimuths of $c$-plane sapphire, repeat every $60^{\circ}$ revealing the symmetry of the $\mathrm{Bi}_{2} \mathrm{Te}_{3}$ and $\mathrm{Sb}_{2} \mathrm{Te}_{3}$ films (space group $R \overline{3} m$ ) [17, 31].

\section{B. X-ray based structural characterization}

X-ray reflectometry (XRR) and x-ray diffraction (XRD) were used to probe the structural properties of the heterostructure samples. Figure 1(c) shows XRD measurements of a representative magnetic TI heterostructure. In the spectrum, the $\left(\begin{array}{lll}0 & 0 & l\end{array}\right)$ reflections are labeled. Note that while Dy-doping of $\mathrm{Bi}_{2} \mathrm{Te}_{3}$ leads to an increased $c$-axis lattice constant (up from $30.43 \AA$ ) [17], Cr-doped $\mathrm{Sb}_{2} \mathrm{Te}_{3}$ has a smaller lattice constant (down from $30.458 \AA$ ), consistent with substitutional doping by a smaller ion (Cr replacing Sb) [31]. Note that Dy doping into $\mathrm{Bi}_{2} \mathrm{Te}_{3}$ is also a substitutional [17]. The presence of superlattice peaks (see inset) is indicative of a wellordered multilayer structure with well-defined interfaces. The bilayer thickness can be estimated to $\sim 8 \mathrm{~nm}$ by measuring the distance between a main Bragg peak and the satellites. The reflections for an undoped $\mathrm{Bi}_{2} \mathrm{Te}_{3} / \mathrm{Sb}_{2} \mathrm{Te}_{3}$ heterostructure are consistent with previously published data on $c$-axis oriented systems [28]. The XRR data [Fig. 1(d)] was fitted using a 10-bilayer model (red curve, simulation), giving $\mathrm{Cr}: \mathrm{Sb}_{2} \mathrm{Te}_{3}$ and $\mathrm{Dy}: \mathrm{Bi}_{2} \mathrm{Te}_{3}$ thicknesses of $3.5 \mathrm{~nm}$ and $3.8 \mathrm{~nm}$, respectively, with roughnesses of around $1 \mathrm{~nm}$. 

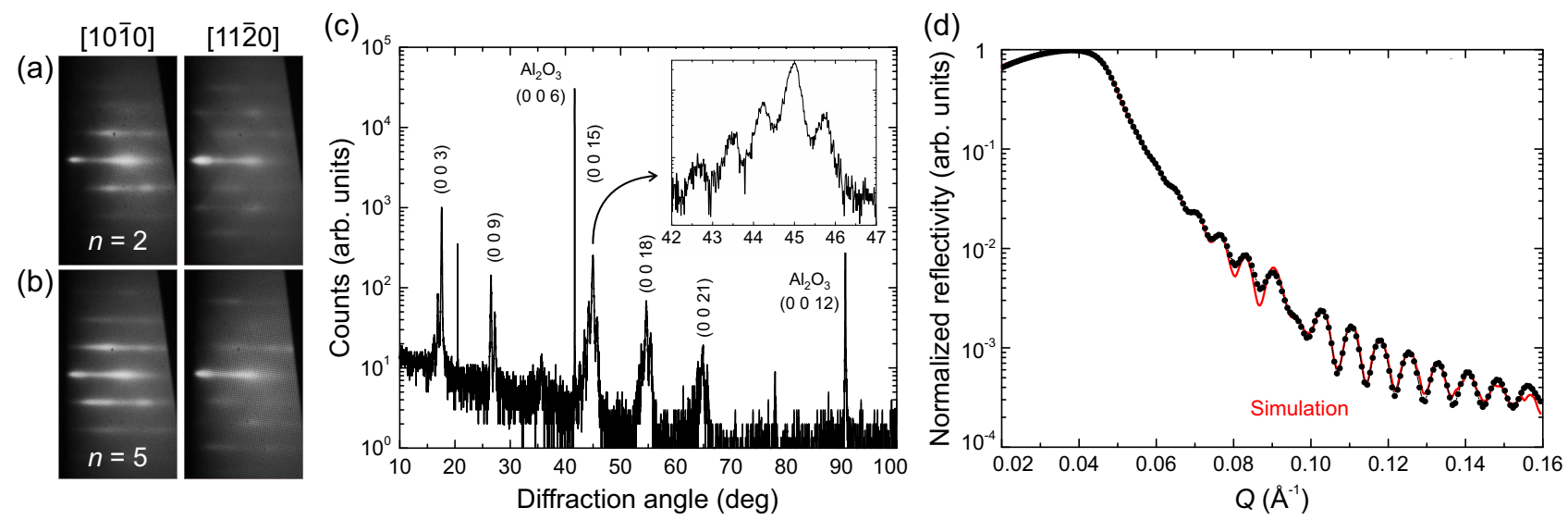

FIG. 1. Structural properties of grown heterostructure. RHEED patterns obtained of the Cr: $\mathrm{Sb}_{2} \mathrm{Te}_{3}$ surface during growth, after (a) $n=2$ and (b) $n=5$ bilayer repeats. Patterns obtained along the [1010] azimuth are shown on the left, and along the

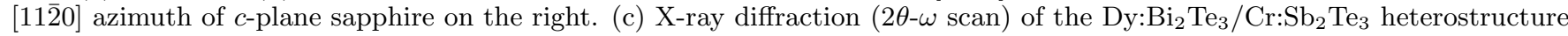
grown on $c$-plane sapphire. The inset shows the superlattice peaks around the (lo 0 15) Bragg peak. (d) X-ray reflectivity scan (black dots), and simulation (red line) as a function of momentum transfer $Q$.

\section{Magnetic spectroscopy}

XAS and XMCD measurements for the heterostructure sample were carried out at the $\mathrm{Cr} L_{2,3}$ and the Dy, Te, and $\mathrm{Sb} M_{4,5}$ edges in total-electron-yield (TEY), with a probing depth of 3-5 nm, and fluorescence-yield and luminescence-yield (LY), which probe the entire heterostructure [34]. The magnetic field was always applied along the x-ray beam. Figure 2(b) shows the $\operatorname{Cr} L_{2,3}$ edges in surface-sensitive TEY mode, carried out at remanence at a temperature of $T=3 \mathrm{~K}$. As reported previously for single-layer $\mathrm{Cr}: \mathrm{Sb}_{2} \mathrm{Te}_{3}$, the $\mathrm{Cr} L_{3}$ shows a double peak structure where the first peak, which is $0.5 \mathrm{eV}$ below the second peak, is more dichroic [45]. Comparison with a $\mathrm{Cr}_{2} \mathrm{O}_{3}$ reference sample demonstrates that the heterostructure surface is not oxidized, which is due to the Bi cap. Oxidation would leads to a $\mathrm{Cr}^{3+}$ peak which is at $1.5 \mathrm{eV}$ higher energy than the predominantly $\mathrm{Cr}^{2+}$ peak found here. The XMCD is in good agreement with previously published data for single-layer Cr-doped $\mathrm{Sb}_{2} \mathrm{Te}_{3}$ [45], where fitting of the spectrum by multiplet calculations [46] gives a local ground state for the $\mathrm{Cr}$ dopant of $\Psi_{g}=0.7 \Psi\left(d^{4} \underline{L}\right)+0.3 \Psi\left(d^{3}\right)$ in an octahedral crystal field of $10 D q=1.5 \mathrm{eV}$, with $\underline{L}$ representing a hole on the adjacent atoms. The overall intensity of the $L_{3}$ and $L_{2}$ peak is negative and positive, respectively, as a result of the $2 p$ core spin-orbit interaction. The $\mathrm{Cr} L_{2,3}$ edges largely coincide with Te $M_{4,5}$ resulting in a sloping background of the XAS, which in the XMCD almost exactly cancels out, apart from a small negative peak at $\sim 573 \mathrm{eV}$ and positive peak at $583 \mathrm{eV}$, characteristic of Te $M_{4,5}$. The Te $M_{5}$ edge shows a negative XMCD signal, just as the $\mathrm{Cr} L_{3}$ edge. Since the azimuthal quantum numbers for the orbitals in these two electric-dipole transitions are opposite ( $\mathrm{Te} 3 d \rightarrow 5 p$ and $\mathrm{Cr} 2 p \rightarrow 3 d$, respectively), this means that the $\mathrm{Te}$ and $\mathrm{Cr}$ moments are antiparallel [34, 45].
Figure 2(c) shows the $\mathrm{Cr} L_{2,3}$ edges at remanence at $T=1.6 \mathrm{~K}$ as measured in LY mode, which averages over the entire heterostructure. The LY spectra retain all of the characteristics discussed above for the TEY spectra, demonstrating the homogeneity of the heterostructure. Note that for practical reasons the TEY measurement was carried out in a different end station on a different sample.

Figure 2(d) shows the LY spectra at the Dy $M_{5}$ edge, taken in remanence at $T=1.6 \mathrm{~K}$. The XAS is in good agreement with the characteristic shape for $\mathrm{Dy}^{3+}$, which is composed of three sharp peaks which are each spaced $\sim 2 \mathrm{eV}$ apart [47]. The spectra for left-circular and rightcircular polarization, $I_{L}$ and $I_{R}$ respectively, are distinctly different, as previously reported for single-layer $\mathrm{Dy}: \mathrm{Bi}_{2} \mathrm{Te}_{3}$ [17, 48]. At remanence there is a small but clear-cut XMCD signal, which suggests that the Dy in the heterostructure has ferromagnetic order at $1.6 \mathrm{~K}$. The integrated Dy $M_{5}$ XMCD has the same sign as the $\mathrm{Cr}$ $L_{3}$, which indicates that the averaged Dy moments are aligned along the direction of the $\mathrm{Cr}$ moments. For the spectra in Fig. 2(d) we obtain an asymmetry at the Dy $M_{5}$ peak maximum equal to $A_{\exp } \equiv\left(I_{L}-I_{R}\right) /\left(I_{L}+I_{R}\right)=$ -0.025 . Comparing this to the theoretical value $A_{\text {Hund }}=$ -1.217 calculated for the Hund's rule ground state $\mathrm{Dy}^{3+}$ $4 f^{9}\left({ }^{6} H_{15 / 2}\right)$ with an effective $4 f$ magnetic moment $\mu_{\text {eff }}$ $=10.65 \mu_{\mathrm{B}}$ /atom [48], we obtain for the heterostructure $\mu_{\mathrm{eff}}=0.22 \mu_{\mathrm{B}} / \mathrm{Dy}$ atom $\left(\sim 2 \%\right.$ of the $A_{\mathrm{Hund}}$ value $)$ at $1.6 \mathrm{~K}$ in remanence. The Dy was also measured in TEY but the signal was very weak since it was concealed by the $\mathrm{Cr}: \mathrm{Sb}_{2} \mathrm{Te}_{3}$ layer on top.

Figure 2(e) shows the XAS and XMCD measurements in $\mathrm{LY}$ at the $\mathrm{Sb} M_{4,5}$ edges in an applied field of $6 \mathrm{~T}$ at $T=3 \mathrm{~K}$. The XMCD shows sharp peaks indicating an induced magnetic Sb moment, which is a hallmark of ferromagnetically ordered single-layer $\mathrm{Cr}: \mathrm{Sb}_{2} \mathrm{Te}_{3}$ [45]. Since the $M_{5}$ is positive and $M_{4}$ is negative for the $\mathrm{Sb}$ 

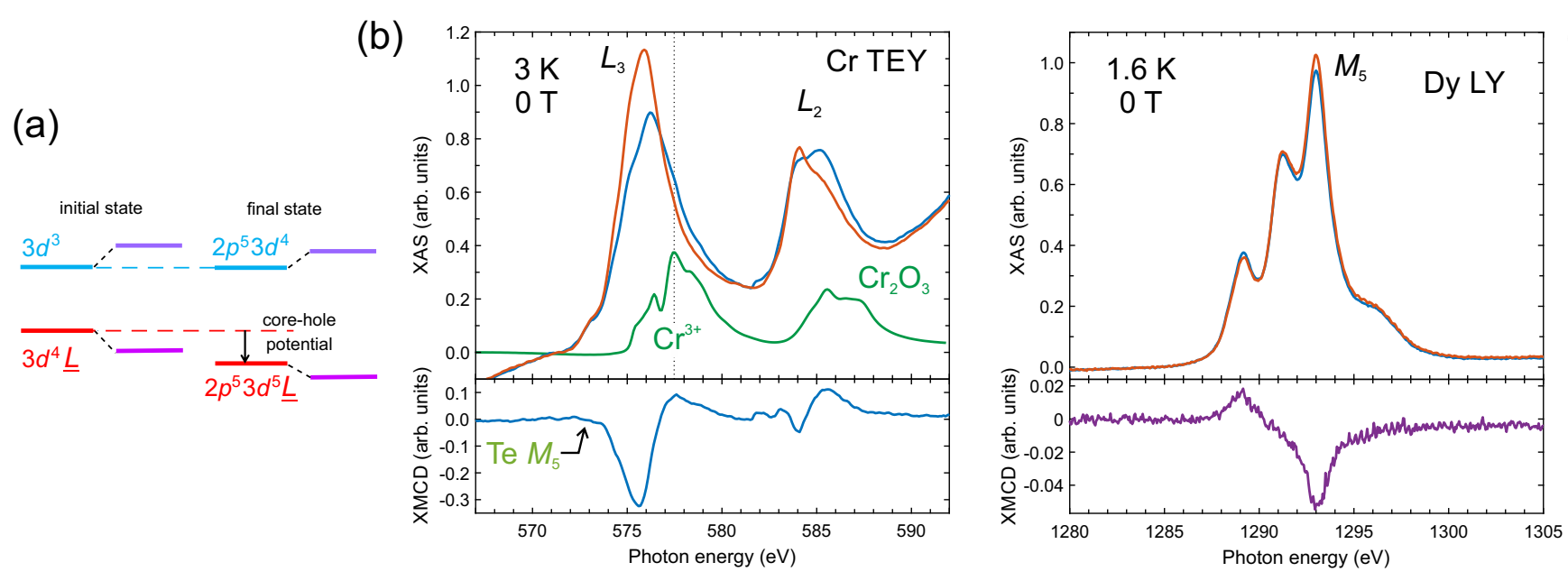

(d)

(c)
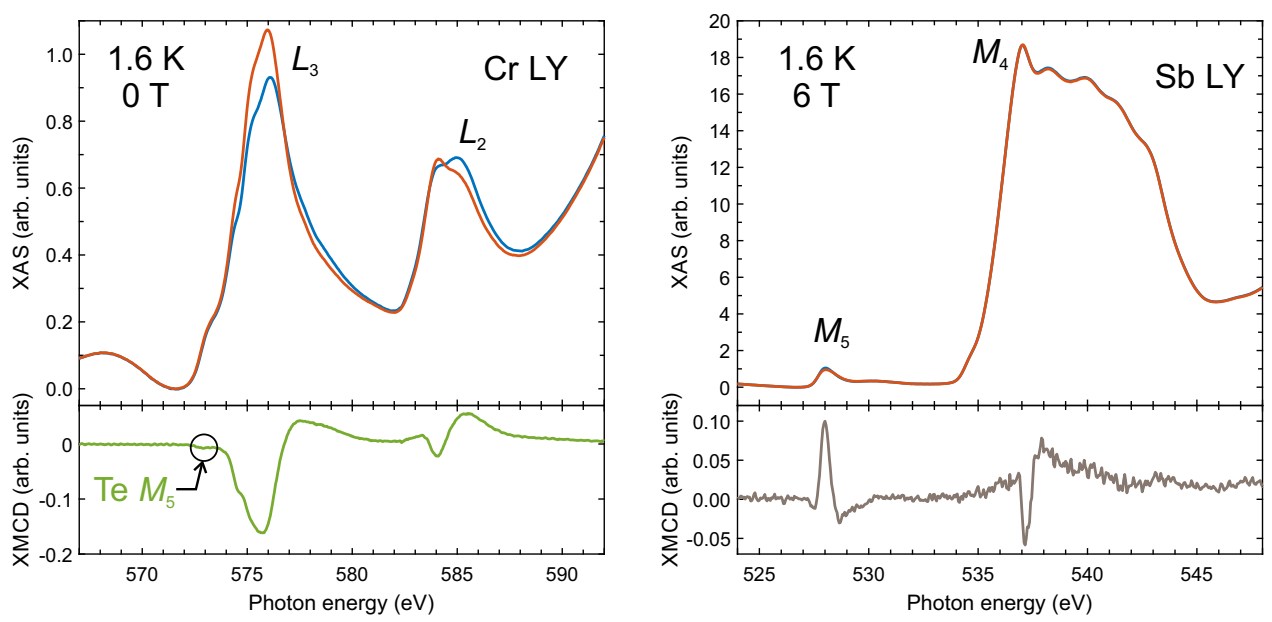

(e)

FIG. 2. XAS and XMCD spectra of $\mathrm{Dy}: \mathrm{Bi}_{2} \mathrm{Te}_{3} / \mathrm{Cr}: \mathrm{Sb}_{2} \mathrm{Te}_{3}$ thin film heterostructure. The red (blue) XAS spectra were measured with right (left) circular polarization. (a) Schematics of the configuration interaction for the Cr dopant showing the initial- and final-state energy levels in the XAS transition. The $3 d^{3}$ and $3 d^{4} \underline{L}$ states are strongly hybridized (mixed), where the $\underline{L}$ is a hole on the ligands. In the final state, the $2 p^{5} 3 d^{5} \underline{L}$ state is lowered in energy by the core-hole potential with respect to the $2 p^{5} d^{4}$ state. In the picture, the energy splittings due to Coulomb and exchange interactions, spin-orbit splitting, and crystal-field interaction are omitted, but are all included in the calculation on an equal basis. (b) Test of the $\mathrm{Bi}$ cap. Cr $L_{2,3} \mathrm{XAS}$ and XMCD in remanence at $3 \mathrm{~K}$, measured in surface-sensitive TEY. Owing to the Bi cap, the spectra do not show a Cr ${ }^{3+}$ oxide peak, which would be $1.5 \mathrm{eV}$ above the predominantly $\mathrm{Cr}^{2+}$ peak (as seen from the $\mathrm{Cr}_{2} \mathrm{O}_{3} \mathrm{XAS}$ reference (green)). A small negative peak in the XMCD at $573 \mathrm{eV}$ is indicative of the Te $M_{5}$ edge. (c) $\mathrm{Cr} L_{2,3} \mathrm{XAS}$ and XMCD in remanence at $1.6 \mathrm{~K}$, measured in LY. (d) Dy $M_{5}$ XAS and XMCD in remanence at $1.6 \mathrm{~K}$, measured in LY. (e) Sb $M_{4,5}$ XAS and XMCD at $1.6 \mathrm{~K}$ in a field of $6 \mathrm{~T}$, measured in LY. The $\sim 8 \mathrm{eV}$ wide absorption structure, which onset coincides with the $\mathrm{Sb} M_{4}$ peak, originates from the $\mathrm{O} K$ edge of the sapphire substrate that is visible in LY but absent in TEY.

$3 d \rightarrow 5 p$ transitions, which is opposite in sign to the $L_{3}$ and $L_{2}$, respectively, of the $\mathrm{Cr} 2 p \rightarrow 3 d$ transitions, we can conclude that the $\mathrm{Sb}$ moments point along the direction of the Cr moments. This is consistent with a hybridization between the $\mathrm{Cr} 3 d$ and $\mathrm{Sb} 5 p$ states.

Element-specific XMCD hysteresis loops [34] were obtained at $T=1.6 \mathrm{~K}$ for both $\mathrm{Cr}$ and Dy by sweeping the applied field with the photon energy tuned to the $\mathrm{Cr} L_{3}$ and Dy $M_{5}$ peak maxima. Figure 3(a) shows the hysteresis loops measured in LY. The loops measured in TEY are similar in shape but noisier and therefore not included here. Figure 3(a) shows that the Cr moment saturates at a field of $3 \mathrm{~T}$. The Dy hysteresis loops are shown in Fig- ure 3(b). The maximum field available for the LY measurements was insufficient to saturate the Dy moments, which saturate at $\sim 7 \mathrm{~T}$. In single-layer $\mathrm{Dy}: \mathrm{Bi}_{2} \mathrm{Te}_{3}$ measurements [17], on the other hand, the saturation magnetization is only $\sim 4 \mathrm{~T}$, which means that the presence of the $\mathrm{Cr}: \mathrm{Sb}_{2} \mathrm{Te}_{3}$ strongly influences its magnetic behavior.

In order to accurately determine the Curie temperature, $T_{\mathrm{C}}$, for each magnetic layer type separately, we employed the Arrott criterion [49]. As a measure for the element-specific magnetization, $M$, as a function of the applied field $H$, we use the XMCD peak asymmetries measured at the respective edges [50]. This method gives a reliable way to determine the $T_{\mathrm{C}}$ by minimiz- 
(a)

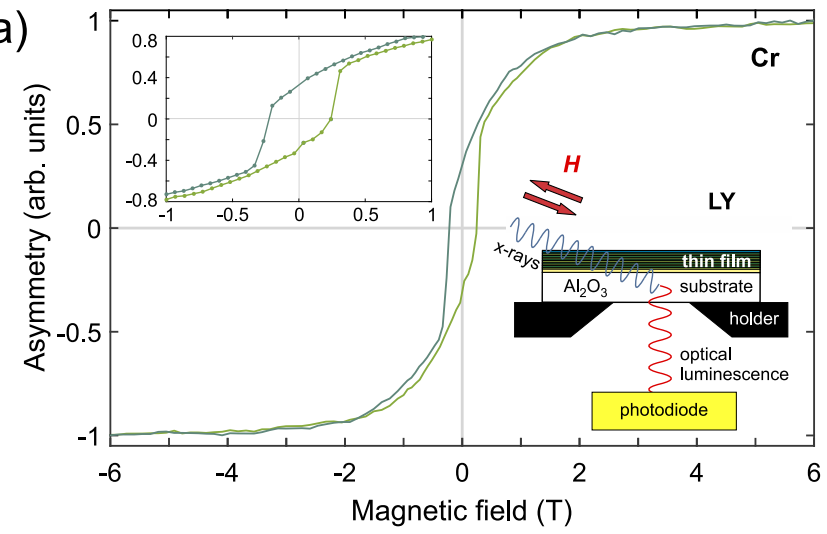

(b)

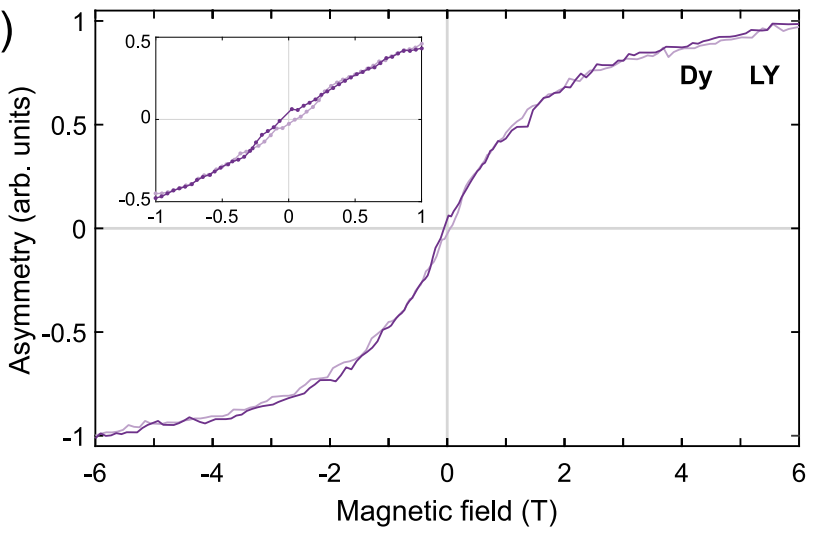

FIG. 3. Hysteresis loops for Cr and Dy. (a) Cr $L_{3}$ and (b) Dy $M_{5}$ hysteresis loops at $3 \mathrm{~K}$, measured in LY. The leftside insets show the low-field behavior. Whereas $\mathrm{Cr}$ shows a loop opening with a coercive field of $\sim 230 \mathrm{mT}$, the Dy loop appears to be closed. Note that the Dy magnetization has not reached saturation at $6 \mathrm{~T}$ (its saturation field is $\sim 7 \mathrm{~T}$ ). The incident angle of the $\mathrm{x}$-rays was $21^{\circ}$ and the applied magnetic field was along the beam, as illustrated in the inset, which shows the geometry for the luminescence yield detection.

ing magnetic contributions from multidomain states and magnetic anisotropies. Arrott plots consist of isotherms of $M^{2}$ vs. $H / M$ plots. At magnetic fields that are large enough to overcome stray fields and domain states, the Arrott plot will show a straight line for each isotherm. Such a behavior is representative of a second-order ferromagnetic phase transition within the framework of meanfield theory. At lower applied fields, disorder in the system will lead to a bending of the isotherms [51]. Figure 4(a) shows the Arrott plot with $M$ obtained from the Cr $L_{3}$ XMCD peak asymmetry in TEY. Parallel straight lines with matching gradients are fitted to the high field region in order to determine the $H / M$ intercepts, which are positive for temperatures above $T_{\mathrm{C}}$ and negative for those below it. The $T_{\mathrm{C}}$ can be determined as the temperature at which the isotherm passes through the origin, i.e., where $M^{2}=0$. Figure 4(b) shows the collection of $H / M$ intercepts for the different temperatures, demonstrating a $T_{\mathrm{C}}$ of $(70 \pm 2) \mathrm{K}$ for the $\mathrm{Cr}$ dopants. This value matches closely to that of single-layer $\mathrm{Cr}: \mathrm{Sb}_{2} \mathrm{Te}_{3}$ of similar doping concentration [31].

Figure 4(c) shows the Arrott plot measured using the background-corrected Dy $M_{5}$ XMCD asymmetry in FY. In this case, a fit with a global slope was not possible, owing to disorder [51]. Instead, linear fits with varying slopes above and below the transition temperature were carried out. The $T_{\mathrm{C}}$ for the Dy is found to be $(17 \pm 1) \mathrm{K}$, which is much different from the paramagnetic or antiferromagnetic behavior in single-layers and bulk crystals of rare earth-doped $\mathrm{Bi}_{2} \mathrm{Te}_{3}[17,24,25]$.

In order to further understand the coupling mechanism between the different layers in the heterostructure, possibly leading to a change in magnetic anisotropy, angulardependent XMCD measurements at the $\mathrm{Cr} L_{3}$ edges were carried out ( $T=3 \mathrm{~K}$, zero applied field). Single-layer $\mathrm{Cr}: \mathrm{Sb}_{2} \mathrm{Te}_{3}$ and $\mathrm{Dy}: \mathrm{Bi}_{2} \mathrm{Te}_{3}$ are expected to have their easy axis out-of-plane and in-plane, respectively.

Figure 5 shows the $\mathrm{Cr} L_{3}$ XMCD peak asymmetry in TEY at remanence as a function of the incidence angle $\theta$ of the x-ray beam. The x-ray helicity vector $P$ is along the beam direction, as shown in the inset. The XMCD asymmetry is proportional to the projection of the $\mathrm{Cr}$ magnetization $M$ onto $P$. The blue dots represent the data points for $\mathrm{Cr}$ obtained in remanence at $3 \mathrm{~K}$, which were fitted with a sine function (red curve). Thus the angular dependent XMCD shows that the averaged $\mathrm{Cr}$ moments have an out-of-plane easy magnetization direction. This demonstrates that the orientation of the magnetic anisotropy for the $\mathrm{Cr}$ moments is not affected by the incorporation in a heterostructure. The complementary Dy measurement in TEY, on the other hand, showed no clear anisotropy within the experimental error bar.

\section{DISCUSSION}

In order to improve the understanding of the coupling effect between different types of layers, theoretical calculations were performed. In the case of heterostructures of ferromagnetically doped TIs and (anti)ferromagnetic materials, where enhanced proximity coupling was able to introduce interfacial spin textures [50,52-54], a deeper understanding has been reached by a combination of theoretical modeling and experiment. Here, to elucidate the obtained experimental results, we performed firstprinciples calculations for the electronic and magnetic structure of $\mathrm{Cr}: \mathrm{Sb}_{2} \mathrm{Te}_{3}$ and $\mathrm{Dy}: \mathrm{Bi}_{2} \mathrm{Te}_{3}$ single-layer films and their interfaces. The magnetic properties were studied within the Heisenberg model, in which the entering exchange parameters $J_{0 j}$, describing the magnetic interaction between magnetic moments, were determined from first-principles using the magnetic force theorem as it is implemented within the multiple scattering theory [43]. The calculated $J_{0 j}$ in the studied systems are presented in Fig. 6, which shows the dependence of the exchange parameters on the interatomic distances. A positive value of $J_{0 j}$ means ferromagnetic coupling, while a negative sign indicates antiferromagnetic interaction. 

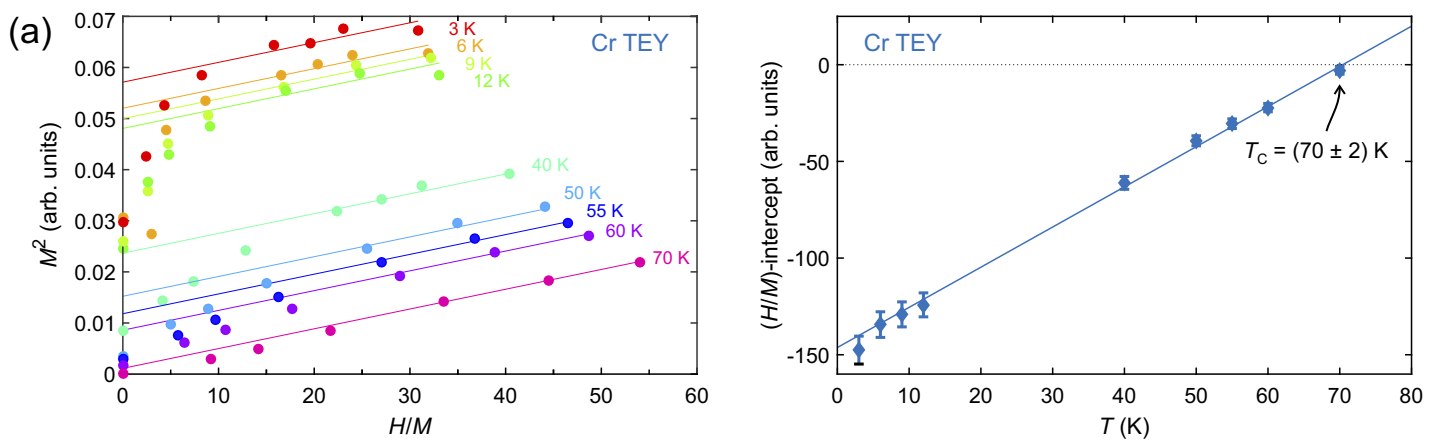

(b)

(c)

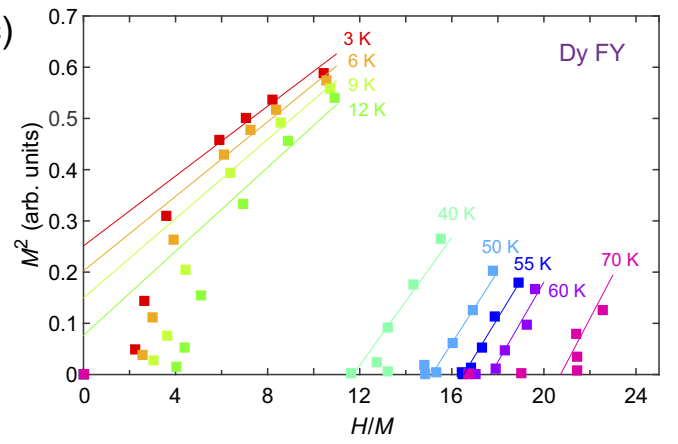

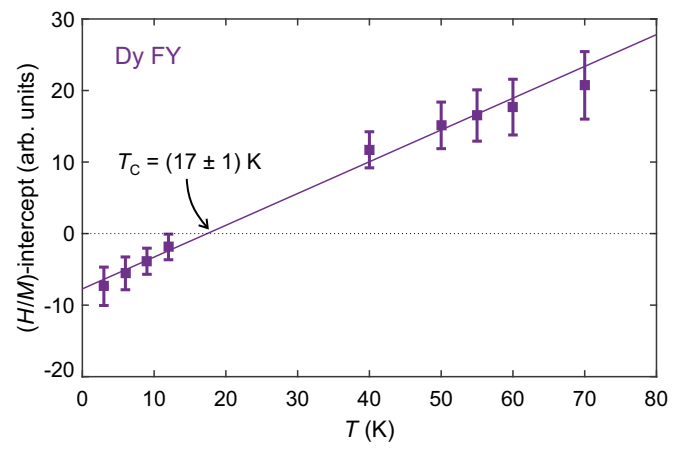

(d)

FIG. 4. Arrott plot analysis of magnetic transition temperatures. Arrott $M^{2}$ vs $H / M$ plots where the magnetization $M$ for a field $H$ is obtained from the XMCD peak asymmetry of the (a) $\mathrm{Cr} L_{3}$ and (b) Dy $M_{5}$ edges, respectively. The error bars are smaller than the symbols representing the data points. The solid lines are linear fits to the high-field data points for each isotherm. The temperature dependence of the $H / M$-intercept allows for the determination of the magnetic transition temperatures of $\mathrm{Cr}$ and $\mathrm{Dy}$, and thus the chemically distinct layers in the heterostructure. For $\mathrm{Cr}$, the $T_{\mathrm{C}}$ is $70 \mathrm{~K}$ (b) and for

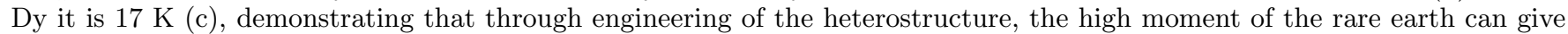
long-range magnetic order through the presence of the Cr-doped layer.

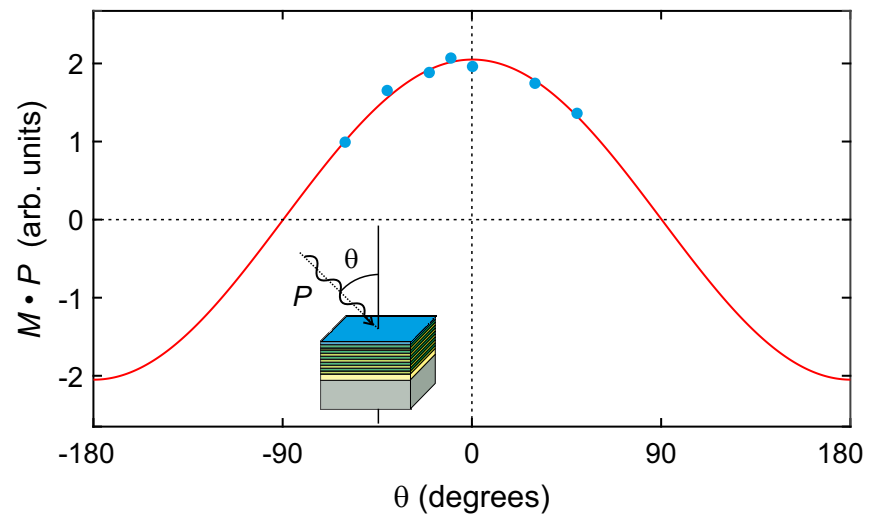

FIG. 5. Magnetic anisotropy of the Cr dopants at remanence. Cr $L_{3}$ XMCD peak asymmetry measured in TEY in zero field as a function of the incidence angle $\theta$ of the beam with respect to the surface normal, as shown in the inset. The x-ray helicity vector $P$ is along the beam direction. The XMCD asymmetry is proportional to the projection of the $\mathrm{Cr}$ magnetization $M$ onto $P$. The blue dots represent the data points for $\mathrm{Cr}$ obtained in remanence at $3 \mathrm{~K}$, which are fitted with a sine function (red curve). The angular behavior shows that the easy direction of the Cr moments is along the surface normal. Instead, no clear angular dependence could be observed for the Dy moments in TEY.
First, the magnetic interaction in a $\mathrm{Cr}: \mathrm{Sb}_{2} \mathrm{Te}_{3}$ singlelayer film is predominantly positive [Fig. 6(a), blue line], implying a relatively strong ferromagnetic coupling between the $\mathrm{Cr}$ moments. The estimated Curie temperature was found to be $102 \mathrm{~K}$, which is higher than the experimental value determined in the current experiment $(70 \mathrm{~K})$. The discrepancy between theory and experiment can be attributed to a presence of various defects such as vacancies and other imperfections in real materials, which can substantially reduce $T_{\mathrm{C}}$. A strong impurity state at the Fermi level in the majority spin channel [see the density of state (DOS) shown in Fig. 7(a)] suggests the Zener type of exchange interaction in this system $[55,56]$. On the other hand, in a $\mathrm{Dy}: \mathrm{Bi}_{2} \mathrm{Te}_{3}$ singlelayer, holes are formed almost equally in both spin channels [Fig. 7(b)], which leads to paramagnetism in this system. The exchange interaction between Dy moments is positive and very strong but only within the Bi layer between the nearest neighbors. The magnetic coupling between different layers is very small and can not force any magnetic order. Nevertheless, according to our simulations, each Dy:Bi layer is ferromagnetic with $T_{\mathrm{C}}=5 \mathrm{~K}$. However, the absence of magnetic coupling between the layers destroys any magnetic order in the system as a whole: the ferromagnetic layers are frustrated. This picture changes at a $\mathrm{Cr}: \mathrm{Sb}_{2} \mathrm{Te}_{3} / \mathrm{Dy}: \mathrm{Bi}_{2} \mathrm{Te}_{3}$ interface. The 

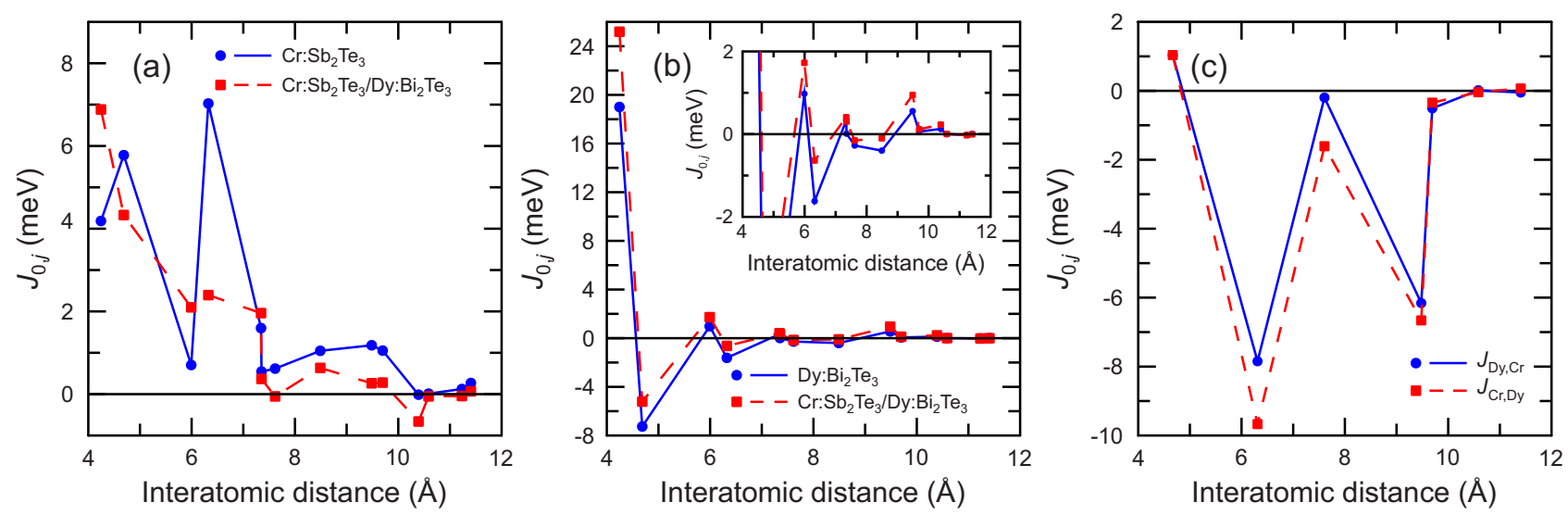

FIG. 6. Exchange coupling between the magnetic moments in single-layer Cr: $\mathrm{Sb}_{2} \mathrm{Te}_{3}, \mathrm{Dy}: \mathrm{Bi}_{2} \mathrm{Te}_{3}$ films and across their interface. The exchange constant $J_{0 j}$ in (a) $\mathrm{Cr}: \mathrm{Sb}_{2} \mathrm{Te}_{3}$ and (b) $\mathrm{Dy}: \mathrm{Bi}_{2} \mathrm{Te}_{2}$ for single layers (blue color) and at the $\mathrm{Cr}_{2} \mathrm{Sb}_{2} \mathrm{Te}_{3} / \mathrm{Dy}: \mathrm{Bi}_{2} \mathrm{Te}_{3}$ interface (red color). The inset in (b) shows the long-range magnetic interaction on an expanded scale. (c) Coupling between Dy and Cr moments across the interface: blue line gives the interaction between Dy moment in the interfacial layer and Cr moments in $\mathrm{Sb}_{2} \mathrm{Te}_{3}$ and red line gives the interaction between Cr moment at the interface and $\mathrm{Dy}$ moments in $\mathrm{Bi}_{2} \mathrm{Te}_{3}$.

hole in the majority spin channel of $\mathrm{Cr}: \mathrm{Sb}_{2} \mathrm{Te}_{3}$ polarizes the hole in $\mathrm{Dy} \mathrm{Bi}_{2} \mathrm{Te}_{3}$ [Fig. $7(\mathrm{~d})$ ] and leads to an enhancement of the magnetic interaction in the vicinity of the interface. First, the magnetic coupling between Dy and $\mathrm{Cr}$ moments is strong and negative, indicating antiparallel alignment of the moments [Fig. 6(c)]. Second, the exchange interaction extends over several layers: a $\mathrm{Cr}$ moment at the interface interacts strongly with Dy moments in the first $\left(J_{\mathrm{Cr}, \mathrm{Dy}}=-9.6 \mathrm{eV}\right)$ and the second $\left(J_{\mathrm{Cr}, \mathrm{Dy}}=-6.1 \mathrm{eV}\right)$ neighboring layers, forcing them to be antiparallel to the $\mathrm{Cr}$ moments, but parallel to each other. Moreover, the proximity with $\mathrm{Cr}: \mathrm{Sb}_{2} \mathrm{Te}_{3}$ enhances the amount of almost free electrons in $\mathrm{Dy}: \mathrm{Bi}_{2} \mathrm{Te}_{3}$, which leads to a stronger indirect exchange interaction between the Dy $4 f$ electrons via the conduction electrons [Ruderman-Kittel-Kasuya-Yosida (RKKY)]: the corresponding exchange parameters are enhanced on the longrange scale [see inset in Fig. 6(b)]. The estimated Curie temperature of $\mathrm{Dy}: \mathrm{Bi}_{2} \mathrm{Te}_{3}$ in the vicinity of the interface was found to be $23 \mathrm{~K}$, which is very close the experimentally obtained value of $17 \mathrm{~K}$.

The proximity to $\mathrm{Dy}: \mathrm{Bi}_{2} \mathrm{Te}_{3}$ also affects the magnetic interaction in $\mathrm{Cr}: \mathrm{Sb}_{2} \mathrm{Te}_{3}$. The coupling between $\mathrm{Cr}$ moments in the film and the Dy moment at the interface, $J_{\mathrm{Dy}, \mathrm{Cr}}$, is almost the same as in the previous case, $J_{\mathrm{Cr} \text {, Dy }}$ [Fig. 6(c)], but the interaction between the $\mathrm{Cr}$ moments is larger [Fig. 6(b), red line]. Especially the interaction between the second nearest layers is enhanced. However, the Curie temperature of the $\mathrm{Cr}: \mathrm{Sb}_{2} \mathrm{Te}_{3}$ layer increases only by $19 \mathrm{~K}$, which is not observed in the current experiment (possibly because of a presence of defects or other imperfections).

In the calculation, the $\mathrm{Cr}: \mathrm{Sb}_{2} \mathrm{Te}_{3}$ layer has out-ofplane magnetization, which is indeed confirmed by the angular dependent measurement of the XMCD. For the $\mathrm{Dy}: \mathrm{Bi}_{2} \mathrm{Te}_{3}$ layer the magnetization is in-plane. At the interface the antiferromagnetic interaction between $\mathrm{Cr}$ and

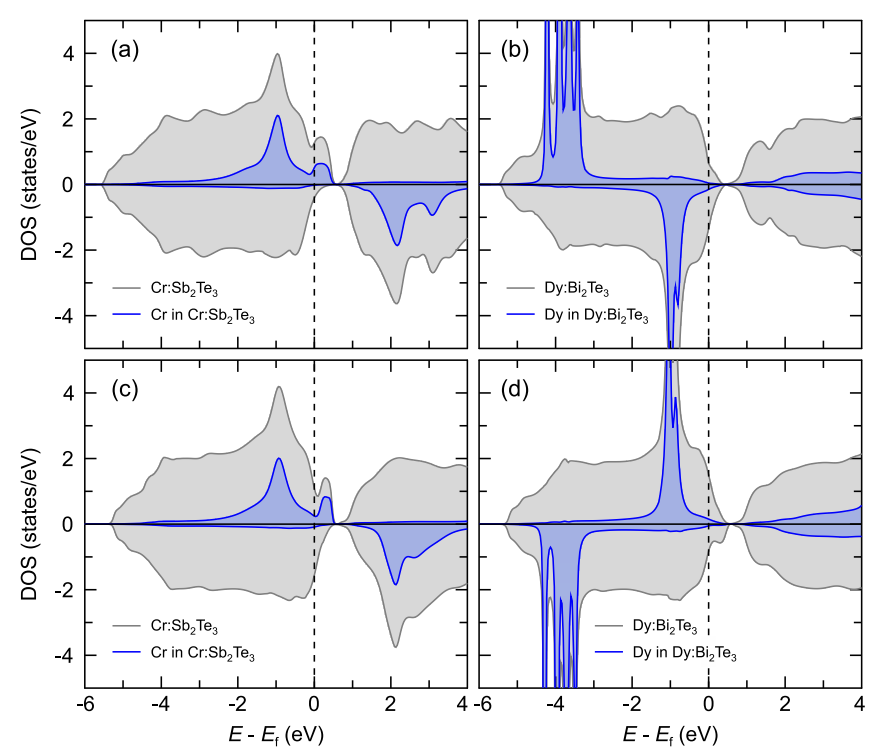

FIG. 7. Calculated densities of states. Single-layer films of (a) $\mathrm{Cr}: \mathrm{Sb}_{2} \mathrm{Te}_{3}$, (b) Dy: $\mathrm{Bi}_{2} \mathrm{Te}_{3}$ and at the $\mathrm{Cr}: \mathrm{Sb}_{2} \mathrm{Te}_{3} / \mathrm{Dy}: \mathrm{Bi}_{2} \mathrm{Te}_{3}$ interface for (c) Cr: $\mathrm{Sb}_{2} \mathrm{Te}_{3}$ and (d) $\mathrm{Dy}: \mathrm{Bi}_{2} \mathrm{Te}_{3}$ layers. The contribution of the magnetic atoms is shown in dark blue color. Positive (negative) ordinates correspond to the spin up (down) DOS.

Dy is very strong and dominated by Cr, so that both layers have out-of-plane magnetization. However, for the Dy moments further away from the interface the situation can be different, similar to the situation in heterostructures of the (in-plane) antiferromagnet $\mathrm{CrSb}$ and (out-ofplane) ferromagnetically ordered Cr-doped $(\mathrm{Bi}, \mathrm{Sb})_{2} \mathrm{Te}_{3}$ [52]. According to the measured XMCD the averaged Dy moment of the overall layer is aligned along the direction of the $\mathrm{Cr}$ moment. 


\section{SUMMARY AND CONCLUSIONS}

In summary, Dy-doped $\mathrm{Bi}_{2} \mathrm{Te}_{3}$ films show a surprisingly high ferromagnetic ordering temperature of $\sim 17 \mathrm{~K}$, when incorporated into a heterostructure with a $\mathrm{Cr}: \mathrm{Sb}_{2} \mathrm{Te}_{3}$ film that has a high $T_{\mathrm{C}}$. The MBE-grown heterostructures are of high crystalline quality, with well-defined interfaces. Our comprehensive x-ray spectroscopy study unambiguously shows that the Dy-doped films are ferromagnetically ordered at remanence. By comparing surface and bulk-sensitive XAS and XMCD we can conclude that the magnetic behavior is reproducible throughout the stack. The Arrott plots using XMCD precisely determine the $T_{\mathrm{C}}$ of both the Dy and Cr within the heterostructure. The significant enhancement of the $T_{\mathrm{C}}$ of $\mathrm{Dy}: \mathrm{Bi}_{2} \mathrm{Te}_{3}$ due to proximity coupling is a very encouraging finding, which has been fully explained by our first-principles calculations. Whereas a single layer of $\mathrm{Cr}: \mathrm{Sb}_{2} \mathrm{Te}_{3}$ is strongly ferromagnetic ordered, singlelayer $\mathrm{Dy}: \mathrm{Bi}_{2} \mathrm{Te}_{3}$ is paramagnetic. Nevertheless, the exchange interaction between Dy moments is leading to ferromagnetic order within the layer, while the interlayer coupling is negligible. At a $\mathrm{Cr}: \mathrm{Sb}_{2} \mathrm{Te}_{3} / \mathrm{Dy}: \mathrm{Bi}_{2} \mathrm{Te}_{3}$ interface, Dy and Cr moments couple strongly through holeinduced polarization, resulting in ferromagnetic order in $\mathrm{Dy}: \mathrm{Bi}_{2} \mathrm{Te}_{3}$ in the vicinity of the interface. The theoretically calculated value of $23 \mathrm{~K}$ is close the experimentally obtained value of $17 \mathrm{~K}$. It is clear that the design of TI heterostructures holds the key to progress in the field of QAH-effect physics, paving the way for dissipationless spintronics applications.

\section{ACKNOWLEDGMENTS}

This publication arises from research funded by the John Fell Oxford University Press Research Fund. We acknowledge the Diamond Light Source at Didcot, UK for provision of synchrotron radiation beamtime at beamline I10 under proposal SI-17480 and the CELLS-ALBA Synchrotron at Barcelona, Spain on beamline BL29 (BOREAS) under proposal 2017022051. A.E. thanks the Deutsche Forschungsgemeinschaft (DFG) through the priority program SPP 1666 . L.B.D. was supported by STFC and EPSRC through a Doctoral Training Award, and A.F. by EPSRC (EP/P021190/1).
[1] X.-L. Qi, T. L. Hughes, and S.-C. Zhang, Phys. Rev. B 78, 195424 (2008).

[2] C. X. Liu, X. L. Qi, X. Dai, Z. Fang, and S. C. Zhang, Phys. Rev. Lett. 101, 146802 (2008).

[3] R. Yu, W. Zhang, H.-J. Zhang, S.-C. Zhang, X. Dai, and Z. Fang, Science 329, 61 (2010).

[4] S. Oh, Science 340, 153 (2013).

[5] C.-Z. Chang, J. Zhang, X. Feng, J. Shen, Z. Zhang, M. Guo, K. Li, Y. Ou, P. Wei, L.-L. Wang, Z.-Q. Ji, Y. Feng, S. Ji, X. Chen, J. Jia, X. Dai, Z. Fang, S.-C. Zhang, K. He, Y. Wang, L. Lu, X.-C. Ma, and Q.-K. Xue, Science 340, 167 (2013).

[6] C.-Z. Chang, W. Zhao, D. Y. Kim, H. Zhang, B. A. Assaf, D. Heiman, S.-C. Zhang, C. Liu, M. H. W. Chan, and J. S. Moodera, Nat. Mater. 14, 473 (2015).

[7] J. G. Checkelsky, R. Yoshimi, A. Tsukazaki, K. S. Takahashi, Y. Kozuka, J. Falson, M. Kawasaki, and Y. Tokura, Nat. Phys. 10, 731 (2014).

[8] Y. Jiang, C. Song, Z. Li, M. Chen, R. L. Greene, K. He, L. Wang, X. Chen, X. Ma, and Q.-K. Xue, Phys. Rev. B 92, 195418 (2015).

[9] A. J. Bestwick, E. J. Fox, X. Kou, L. Pan, K. L. Wang, and D. Goldhaber-Gordon, Phys. Rev. Lett. 114, 187201 (2015).

[10] Z. Zhou, Y.-J. Chien, and C. Uher, Phys. Rev. B 74, 224418 (2006).

[11] I. Lee, C. K. Kim, J. Lee, S. J. L. Billinge, R. Zhong, J. A. Schneeloch, T. Liu, T. Valla, J. M. Tranquada, G. Gu, and J. C. S. Davis, Proc. Natl. Acad. Sci. U.S.A 112, 1316 (2015).

[12] A. I. Figueroa, G. van der Laan, L. J. Collins-McIntyre, S.-L. Zhang, A. A. Baker, S. E. Harrison, P. Schönherr, G. Cibin, and T. Hesjedal, Phys. Rev. B 90, 134402
(2014).

[13] A. I. Figueroa, G. van der Laan, L. J. Collins-McIntyre, G. Cibin, A. J. Dent, and T. Hesjedal, J. Phys. Chem. C 119, 17344 (2015).

[14] L. B. Duffy, A. I. Figueroa, G. van der Laan, and T. Hesjedal, Phys. Rev. Materials 1, 064409 (2017).

[15] X. Kou, L. He, M. Lang, Y. Fan, K. Wong, Y. Jiang, T. Nie, W. Jiang, P. Upadhyaya, Z. Xing, Y. Wang, F. Xiu, R. N. Schwartz, and K. L. Wang, Nano Lett. 13, 4587 (2013).

[16] M. Mogi, R. Yoshimi, A. Tsukazaki, K. Yasuda, Y. Kozuka, K. S. Takahashi, M. Kawasaki, and Y. Tokura, Appl. Phys. Lett. 107, 182401 (2015).

[17] S. E. Harrison, L. J. Collins-McIntyre, S.-L. Zhang, A. A. Baker, A. I. Figueroa, A. J. Kellock, A. Pushp, S. S. P. Parkin, J. S. Harris, G. van der Laan, and T. Hesjedal, J. Phys.: Condens. Matter 27, 245602 (2015).

[18] S. E. Harrison, L. J. Collins-McIntyre, P. Schönherr, A. Vailionis, V. Srot, P. A. van Aken, A. J. Kellock, A. Pushp, S. S. P. Parkin, J. S. Harris, B. Zhou, Y. L. Chen, and T. Hesjedal, Sci. Rep. 5, 15767 (2015).

[19] M. Mogi, M. Kawamura, R. Yoshimi, A. Tsukazaki, Y. Kozuka, N. Shirakawa, K. S. Takahashi, M. Kawasaki, and Y. Tokura, Nat. Mater. 16, 516 (2017).

[20] D. Xiao, J. Jiang, J.-H. Shin, W. Wang, F. Wang, Y.-F. Zhao, C. Liu, W. Wu, M. H. W. Chan, N. Samarth, and C.-Z. Chang, Phys. Rev. Lett. 120, 056801 (2018).

[21] R. Dingle, H. L. Störmer, A. C. Gossard, and W. Wiegmann, Appl. Phys. Lett. 33, 665 (1978).

[22] H. L. Störmer, A. Pinczuk, A. C. Gossard, and W. Wiegmann, Appl. Phys. Lett. 38, 691 (1981).

[23] Y. L. Chen, J.-H. Chu, J. G. Analytis, Z. K. Liu, K. Igarashi, H.-H. Kuo, X. L. Qi, S. K. Mo, R. G. Moore, 
D. H. Lu, M. Hashimoto, T. Sasagawa, S. C. Zhang, I. R. Fisher, Z. Hussain, and Z. X. Shen, Science 329, 659 (2010).

[24] S. E. Harrison, L. J. Collins-McIntyre, S. Li, A. A. Baker, L. R. Shelford, Y. Huo, A. Pushp, S. S. P. Parkin, J. S. Harris, E. Arenholz, G. van der Laan, and T. Hesjedal, J. Appl. Phys. 115, 023904 (2014).

[25] S. E. Harrison, L. J. Collins-McIntyre, S. L. Zhang, A. A. Baker, A. I. Figueroa, A. J. Kellock, A. Pushp, Y. L. Chen, S. S. P. Parkin, J. S. Harris, G. van der Laan, and T. Hesjedal, Appl. Phys. Lett. 107, 182406 (2015).

[26] C.-Z. Chang, P. Tang, Y.-L. Wang, X. Feng, K. Li, Z. Zhang, Y. Wang, L.-L. Wang, X. Chen, C. Liu, W. Duan, K. He, X.-C. Ma, and Q.-K. Xue, Phys. Rev. Lett. 112, 056801 (2014).

[27] R. Venkatasubramanian, T. Colpitts, E. Watko, M. Lamvik, and N. ElMasry, J. Cryst. Growth 170, 817 (1997).

[28] Y. Iwata, H. Kobayashi, S. Kikuchi, E. Hatta, and K. Mukasa, J. Cryst. Growth 203, 125 (1999).

[29] M. Lanius, J. Kampmeier, C. Weyrich, S. Kölling, M. Schall, P. Schüffelgen, E. Neumann, M. Luysberg, G. Mussler, P. M. Koenraad, T. Schäpers, and D. Grützmacher, Cryst. Growth Des. 16, 2057 (2016).

[30] L. J. Collins-McIntyre, S. E. Harrison, P. Schönherr, N.-J. Steinke, C. J. Kinane, T. R. Charlton, D. AlbaVeneroa, A. Pushp, A. J. Kellock, S. S. P. Parkin, J. S. Harris, S. Langridge, G. van der Laan, and T. Hesjedal, EPL (Europhys. Lett.) 107, 57009 (2014).

[31] L. J. Collins-McIntyre, L. B. Duffy, A. Singh, N.-J. Steinke, C. J. Kinane, T. R. Charlton, A. Pushp, A. J. Kellock, S. S. P. Parkin, S. N. Holmes, C. H. W. Barnes, G. van der Laan, S. Langridge, and T. Hesjedal, EPL (Europhys. Lett.) 115, 27006 (2016).

[32] S. E. Harrison, S. Li, Y. Huo, B. Zhou, Y.-L. Chen, and J. S. Harris, Appl. Phys. Lett. 102, 171906 (2013).

[33] G. van der Laan, J. Phys. Conf. Ser. 430, 012127 (2013).

[34] G. van der Laan and A. I. Figueroa, Coord. Chem. Rev. 277-278, 95 (2014).

[35] J. Stöhr and H. König, Phys. Rev. B 75, 3748 (1995).

[36] G. van der Laan and H. A. Dürr, Physica B 248, 121 (1988).

[37] J. P. Perdew, K. Burke, and M. Ernzerhof, Phys. Rev. Lett. 77, 3865 (1996).
[38] M. Lüders, A. Ernst, W. M. Temmerman, Z. Szotek, and P. J. Durham, J. Phys. Condens. Matter 13, 8587 (2001).

[39] M. Geilhufe, S. Achilles, M. A. Köbis, M. Arnold, I. Mertig, W. Hergert, and A. Ernst, J. Phys. Condens. Matter 27, 435202 (2015).

[40] P. Soven, Phys. Rev. 156, 809 (1967).

[41] B. L. Gyorffy, Phys. Rev. B 5, 2382 (1972).

[42] V. I. Anisimov, J. Zaanen, and O. K. Andersen, Phys. Rev. B 44, 943 (1991).

[43] A. I. Liechtenstein, M. I. Katsnelson, V. P. Antropov, and V. A. Gubanov, J. Magn. Magn. Mater. 67, 65 (1987).

[44] S. V. Tyablikov, Methods in the quantum theory of magnetism (Plenum Press, New York, 1967).

[45] L. B. Duffy, A. I. Figueroa, L. Gładczuk, N.-J. Steinke, K. Kummer, G. van der Laan, and T. Hesjedal, Phys. Rev. B 95, 224422 (2017).

[46] G. van der Laan and B. T. Thole, Phys. Rev. B 43, 13401 (1991).

[47] B. T. Thole, G. van der Laan, J. C. Fuggle, G. A. Sawatzky, R. C. Karnatak, and J. M. Esteva, Phys. Rev. B 32, 5107 (1985).

[48] A. I. Figueroa, A. A. Baker, S. Harrison, K. Kummer, G. van der Laan, and T. Hesjedal, J. Magn. Magn. Mater. 422, 93 (2017).

[49] A. Arrott and J. E. Noakes, Phys. Rev. Lett. 19, 786 (1967).

[50] A. A. Baker, A. I. Figueroa, K. Kummer, L. J. CollinsMcIntyre, T. Hesjedal, and G. van der Laan, Phys. Rev. B 92, 094420 (2015).

[51] I. Yeung, R. M. Roshko, and G. Williams, Phys. Rev. B 34, 3456 (1986).

[52] Q. L. He, X. Kou, A. J. Grutter, G. Yin, L. Pan, X. Che, Y. Liu, T. Nie, B. Zhang, S. M. Disseler, B. J. Kirby, W. Ratcliff II, Q. Shao, K. Murata, X. Zhu, G. Yu, Y. Fan, M. Montazeri, X. Han, J. A. Borchers, and K. L. Wang, Nat. Mater. 16, 94 (2017).

[53] M. Li, C.-Z. Chang, B. J. Kirby, M. E. Jamer, W. Cui, L. Wu, P. Wei, Y. Zhu, D. Heiman, J. Li, and J. S. Moodera, Phys. Rev. Lett. 115, 087201 (2015).

[54] T. Hesjedal and Y. Chen, Nat. Mater. 16, 3 (2017).

[55] C. Zener, Phys. Rev. 81, 440 (1951).

[56] C. Zener, Phys. Rev. 82, 403 (1951). 